\title{
EXTENSION OF CONTINUOUS MAPPINGS AND H$_{1}$-RETRACTS
}

\author{
OLENA KARLOVA
}

(Received 10 March 2008)

\begin{abstract}
We prove that any continuous mapping $f: E \rightarrow Y$ on a completely metrizable subspace $E$ of a perfect paracompact space $X$ can be extended to a Lebesgue class one mapping $g: X \rightarrow Y$ (that is, for every open set $V$ in $Y$ the preimage $g^{-1}(V)$ is an $F_{\sigma}$-set in $X$ ) with values in an arbitrary topological space $Y$.
\end{abstract}

2000 Mathematics subject classification: primary 54C20, 54C05; secondary 54C15.

Keywords and phrases: extension, Lebesgue class one mapping, continuous function.

\section{Introduction}

A mapping $f: X \rightarrow Y$ from a topological space $X$ to a topological space $Y$ is called $a$ Lebesgue class $\alpha$ mapping (or a mapping of the $\alpha$ th Lebesgue class) if for every closed set $F$ in $Y$ the set $f^{-1}(F)$ is of the multiplicative class $\alpha$ in $X$. The family of all such mappings $f: X \rightarrow Y$ we denote by $H_{\alpha}(X, Y)$. Moreover, we write $f \in H_{1}(X, Y)$ if for every open set $V$ in $Y$ the preimage $g^{-1}(V)$ is an $F_{\sigma}$-set in $X$.

Obviously, if $X$ or $Y$ is a perfect space then any continuous mapping $f: X \rightarrow Y$ is of the first Lebesgue class.

Classification of mappings naturally leads to the problem of the extension of mappings from a subset of a topological space to the whole space with preservation of the mapping class or its estimation. So, such classical results as the Tietze theorem [4, p. 116] or the Dugundji theorem [3] give the possibility of the extension of a continuous mapping to a continuous mapping.

Many mathematicians (F. Hausdorff [8], W. Sierpiński [13], G. Alexits [1], H. Hahn [5], K. Kuratowski [11]) have considered the extension of real-valued functions of some Lebesgue class.

Kuratowski [11] proved that every mapping $f \in H_{\alpha}(E, Y)$ on a subset $E$ of a metric space $X$ with values in a complete metric separable space $Y$ can be extended to a mapping $g: B \rightarrow Y$ of class $\alpha$ such that the set $B \supseteq E$ is of the multiplicative class $\alpha+1$. Moreover, if $E$ is of the multiplicative class $\alpha>0$ then $f$ can be extended to

(c) 2009 Australian Mathematical Society 0004-9727/09 \$A2.00+0.00 
a Lebesgue mapping of class $\alpha$ on the whole space $X$. Consequently, every mapping of the Lebesgue class $\alpha \geq 0$ on a set $E \subseteq X$ can be extended to a mapping of the Lebesgue class $\alpha+1$ on $X$. In particular, the following result holds.

THEOREM 1.1. Let $X$ be a metric space, $Y$ be a complete metric separable space and $E \subseteq X$. Then every continuous mapping $f: E \rightarrow Y$ can be extended to a mapping $g \in H_{1}(X, Y)$.

It follows from the above that the problem of the extension of a continuous function (Lebesgue class one function) to a continuous function (Lebesgue class one function) essentially differs from the problems of the extension of functions preserving their class. For example, if $X=\mathbb{R}$ and $E=\mathbb{Q}$ then not every continuous function $f: E \rightarrow \mathbb{R}$ can be extended to a continuous function defined on $X$; moreover, it is easy to construct an everywhere discontinuous function $f: E \rightarrow \mathbb{R}$ which is of the first Lebesgue class and cannot be extended to a function of the first Lebesgue class on $X$. On the other hand, Theorem 1.1 implies that every continuous function $f: E \rightarrow \mathbb{R}$ can be extended to a function $g: X \rightarrow \mathbb{R}$ of the first Lebesgue class.

In connection with Theorem 1.1 the following question arises.

QUESTION 1.2. Is it possible to omit the assumption of separability on space $Y$ in Theorem 1.1?

Hansell studied the problem of the extension of Lebesgue mappings with nonseparable metrizable ranges using the notion of $\sigma$-discrete mappings as introduced by Stone [14].

Recall that a family $\mathcal{A}$ of subsets of a topological space $X$ is said to be discrete if for every point $x \in X$ there exists a neighborhood $U$ which intersects with at most one set from $\mathcal{A}$.

A family $\mathcal{A}$ is said to be $\sigma$-discrete if it can be written as a countable union of discrete families.

The family $\mathcal{B}$ of subsets of a topological space $X$ is said to be a base for a mapping $f: X \rightarrow Y$ if for every open set $V$ in $Y$ there exists a subfamily $\mathcal{B}_{V} \subseteq \mathcal{B}$ such that $f^{-1}(V)=\cup \mathcal{B}_{V}$. If, moreover, the system $\mathcal{B}$ is $\sigma$-discrete then it is said to be $a \sigma$ discrete base for $f$ and $f$ is said to be $a \sigma$-discrete mapping. The family of all $\sigma$ discrete mappings we denote by $\Sigma(X, Y)$.

Obviously, every mapping with a second countable range space is $\sigma$-discrete. Also it is easy to see that every continuous mapping with metrizable domain or range is $\sigma$-discrete since a metrizable space has a $\sigma$-discrete base [4].

The paper of Hansell [6] mentioned above contains the following result.

THEOREM 1.3 [6, Theorem 9]. Let $X$ be a paracompact space, $Y$ a complete metric space, $E \subseteq X$ and $f: E \rightarrow Y$ a $\sigma$-discrete Lebesgue mapping of class $\alpha$. Then $f$ can be extended to a Lebesgue mapping $g: B \rightarrow Y$ of class $\alpha$ so that the set $B \supseteq E$ is of multiplicative class $\alpha+1$.

The following question naturally arises. 
QUESTION 1.4. Is it possible to replace the set $B$ in Theorem 1.3 with the whole space $X$ ?

The most recent (to the best of our knowledge) result on the extension of Lebesgue functions is due to Kalenda and Spurný.

THEOREM 1.5 [9, Theorem 29]. Let E be a Lindelöf subspace of a completely regular space $X, Y$ a complete metric separable space and

(i) $E$ be hereditarily Baire or

(ii) $E$ be $G_{\delta}$ in $X$.

Then every mapping $f \in H_{1}(E, Y)$ can be extended to a mapping $g \in H_{1}(X, Y)$.

At the same time it is interesting to study when we can extend mappings with values in an arbitrary topological space.

In Section 2 we introduce and study the notion of $H_{1}$-retract which is tightly connected with the problem of the extension of continuous mappings to Lebesgue class one mappings with values in an arbitrary topological space (analogously, the notion of a retract is connected with the extension of continuous mappings with preservation of continuity).

Furthermore, in Section 3 we prove that every continuous mapping $f: E \rightarrow Y$ on a completely metrizable subspace $E$ of a perfect paracompact space $X$ with values in an arbitrary topological space $Y$ can be extended to a Lebesgue class one mapping $g: X \rightarrow Y$. This result implies a positive answer to Question 1.2. In addition, we give a negative answer to Question 1.4.

\section{2. $H_{1}$-retracts and their properties}

Let $X$ be a topological space and $E \subseteq X$. Recall [2] that a set $E$ is said to be $a$ retract of $X$ if there exists a continuous mapping $r: X \rightarrow E$ such that $r(x)=x$ for all $x \in E$. The mapping $r$ is called a retraction of $X$ onto $E$. It is easy to see that a set $E \subseteq X$ is a retract of $X$ if and only if for any topological space $Y$ every continuous mapping $f: E \rightarrow Y$ can be extended to a continuous mapping $g: X \rightarrow Y$.

We call a subset $E$ of a topological space $X$ an $H_{1}$-retract of $X$ if there exists a mapping $r \in H_{1}(X, E)$ such that $r(x)=x$ for all $x \in E$. We call the mapping $r$ an $H_{1}$-retraction of $X$ onto $E$.

The following properties of $H_{1}$-retracts immediately follow from the definition.

Proposition 2.1. Let $X$ be a topological space. A set $E \subseteq X$ is an $H_{1}$-retract of $X$ if and only if for an arbitrary space $Y$ every continuous mapping $f: E \rightarrow Y$ can be extended to a Lebesgue class one mapping $g: X \rightarrow Y$.

Proposition 2.2. Let $E$ be an $H_{1}$-retract of a topological space $X$. Then $E$ is a perfect space.

A subset $A$ of a topological space $X$ is said to be an ambiguous set if $A$ is simultaneously $F_{\sigma}$ and $G_{\delta}$ in $X$. 
Proposition 2.3. Let $X$ be a metrizable space and $E$ be an $H_{1}$-retract of $X$. Then $E$ is $G_{\delta}$ in $X$.

Proof. Let $r: X \rightarrow E$ be an $H_{1}$-retraction of $X$ onto $E$. It is easy to see that $E=\{x \in X \mid r(x)=x\}$.

Consider the diagonal $\Delta=\left\{\left(x^{\prime}, x^{\prime \prime}\right) \in X \times X \mid x^{\prime}=x^{\prime \prime}\right\}$ of the space $X^{2}$ and the mapping $h: X \rightarrow X \times X, h(x)=(r(x), x)$. Since $r \in H_{1}(X, E) \subseteq H_{1}(X, X)$ and the mapping $g: X \rightarrow X, g(x)=x$ is continuous, according to [7, Theorem 1] the mapping $h: X \rightarrow X \times X$ is of the first Lebesgue class. Since $\Delta$ is closed in $X \times X$, the set $E=h^{-1}(\Delta)$ is $G_{\delta}$ in $X$.

Note that an $H_{1}$-retract may, in general, be even a nonmeasurable set. Moreover, the following example shows that the assumption of metrizability of $X$ in the previous proposition is essential.

EXAMPLE 2.4. There exists a nonmeasurable $H_{1}$-retract $E$ of a perfect separable linear ordered compact space $X$.

Proof. Let $X=[0,1] \times\{0,1\}$ be endowed with the lexicographic order, that is $(x, i)<(y, j)$ if $x<y$ or $x=y$ and $i<j, i, j \in\{0,1\}$. Note that $X$ satisfies necessary conditions (see [4, p. 318]).

Consider a set $E=\{(x, 0) \mid x \in[0,1]\}$. A mapping $r: X \rightarrow E, r(x, i)=(x, 0)$, is of the first Lebesgue class.

It remains to prove that $E$ is nonmeasurable.

For a set $A \subseteq X$ denote $A^{+}=\{x \in[0,1] \mid(x, 1) \in A\}$ and $A^{-}=\{x \in[0,1] \mid$ $(x, 0) \in A\}$. It is not hard to prove that for any open or closed set $A$ in $X$ we have $\left|A^{+} \Delta A^{-}\right| \leq \aleph_{0}$. This implies that $\left|B^{+} \Delta B^{-}\right| \leq \aleph_{0}$ for any measurable set $B$. But $E^{+}=\emptyset$ and $E^{-}=[0,1]$. Hence, $E$ is a nonmeasurable set.

Proposition 2.5. Let $X$ and $Y$ be topological spaces, $E$ be an ambiguous subset of $X$ and $f: E \rightarrow Y$ be a Lebesgue class one mapping. Then there exists a Lebesgue class one mapping $g: X \rightarrow Y$ such that $\left.g\right|_{E}=f$.

Corollary 2.6. Let $X$ be a topological space and $E$ be a perfect ambiguous subset of $X$. Then $E$ is an $H_{1}$-retract of $X$.

We call a subset $E$ of a topological space $X a \mathrm{Coz}_{\delta}$-set if there exists a sequence of continuous functions $f_{n}: X \rightarrow[0,1]$ such that $E=\bigcap_{n=1}^{\infty} f_{n}^{-1}((0,1])$. We call the complement to a $\mathrm{Coz}_{\delta}$-set a $\operatorname{Zer}_{\sigma}$-set. We call a set which is simultaneously $\mathrm{Coz}_{\delta}$ and $\operatorname{Zer}_{\sigma}$ a functionally ambiguous set.

Proposition 2.7. Let $E_{1}, \ldots, E_{n}$ be disjoint $H_{1}$-retracts of topological space $X$ and let $E_{i}$ be $\mathrm{Coz}_{\delta}$ in $X$ for every $i \in\{1, \ldots, n\}$. Then the union $E=\bigcup_{i=1}^{n} E_{i}$ is an $H_{1}$-retract of $X$. 
PROOF. First we obtain that for every finite family of disjoint $\mathrm{Coz}_{\delta}$-sets $E_{1}, \ldots, E_{n}$ there exist disjoint functionally ambiguous sets $B_{1}, \ldots, B_{n}$ such that $E_{i} \subseteq B_{i}$ for every $i \in\{1, \ldots, n\}$ and $X=\bigcup_{i=1}^{n} B_{i}$.

Let $n=2$ and $E_{1}, E_{2}$ be disjoint $\operatorname{Coz}_{\delta}$-sets. Then the complements $E_{i}^{c}=X \backslash E_{i}$, $i=1,2$, are $\operatorname{Zer}_{\sigma}$ and $E_{1}^{c} \cup E_{2}^{c}=X$. From [10, Lemma 3.2] it follows that there exist functionally ambiguous sets $B_{1}$ and $B_{2}$ such that $B_{1}^{c} \subseteq E_{1}^{c}, B_{2}^{c} \subseteq E_{2}^{c}, B_{1}^{c} \cup B_{2}^{c}=X$ and $B_{1}^{c} \cap B_{2}^{c}=\emptyset$. Then $E_{1} \subseteq B_{1}, E_{2} \subseteq B_{2}, B_{1} \cap B_{2}=\emptyset$ and $B_{1} \cup B_{2}=X$.

Let $n>2$ and the assumption holds when we have $n-1$ sets. There exist disjoint functionally ambiguous sets $\widetilde{B}_{1}, \ldots, \widetilde{B}_{n-1}$ such that $E_{i} \subseteq \widetilde{B}_{i}$ if $1 \leq i \leq n-2$, $E_{n-1} \cup E_{n} \subseteq \widetilde{B}_{n-1}$ and $X=\bigcup_{i=1}^{n-1} \widetilde{B}_{i}$. Moreover, there exist disjoint functionally ambiguous sets $C$ and $D$ such that $E_{n-1} \subseteq C, E_{n} \subseteq D$ and $C \cup D=X$. Set $B_{i}=\widetilde{B}_{i}$ for $i=1, \ldots, n-2, B_{n-1}=\widetilde{B}_{n-1} \cap C$ and $B_{n}=\widetilde{B}_{n-1} \cap D$.

Let $r_{i}: X \rightarrow E_{i}$ be $H_{1}$-retractions, $1 \leq i \leq n$. For every $x \in X$ define $r(x)=r_{i}(x)$ if $x \in B_{i}$ for some $i \in\{1, \ldots, n\}$. Clearly, $r \in H_{1}(X, E)$ and $r(x)=x$ if $x \in E$.

\section{Extension of continuous mappings to the first class mappings from completely metrizable subspaces}

In this section we prove the main results of this paper. All topological spaces will be considered to be Hausdorff.

We say that a family $\mathcal{A}=\left(A_{i}: i \in I\right)$ of sets $A_{i}$ refines a family $\mathcal{B}=\left(B_{j}: j \in J\right)$ of sets $B_{j}$ if for every $i \in I$ there exists $j \in J$ such that $A_{i} \subseteq B_{j}$. We write this as $\mathcal{A} \preceq \mathcal{B}$.

LEMMA 3.1. Let $X$ be a perfect paracompact space and $\mathcal{G}$ be a locally finite cover of $X$ by ambiguous sets. Then there exists a disjoint locally finite cover of $X$ by ambiguous sets which refines $\mathcal{G}$.

PROOF. Without loss of generality we may assume that $\mathcal{G}=\left\{G_{\alpha} \mid 0 \leq \alpha<\beta\right\}$, where $\beta$ is some ordinal.

Denote $A_{0}=G_{0}$. For every $0<\alpha<\beta$ let $A_{\alpha}=G_{\alpha} \backslash \bigcup_{\xi<\alpha} G_{\xi}$. According to Michael's theorem [4, p. 430], the set $\bigcup_{\xi<\alpha} G_{\xi}$ is ambiguous as a locally finite union of ambiguous sets. Then the set $A_{\alpha}$ is also ambiguous. Clearly, the family $\mathcal{A}=\left(A_{\alpha}: 0 \leq \alpha<\beta\right)$ is to be found.

The next theorem is the main result of our paper.

THEOREM 3.2. Let $X$ be a perfect paracompact space and $E \subseteq X$ be a completely metrizable subspace of $X$. Then $E$ is an $H_{1}$-retract of $X$.

PROOF. Let $d$ be a metric on $E$ such that $(E, d)$ is a complete metric space and $d$ induces the topology in $E$.

For every $n \in \mathbb{N}$ consider a cover $\mathcal{V}_{n}$ of the set $E$ by open balls with radius $1 /\left(2^{n+2}\right)$. For every ball $V \in \bigcup_{n=1}^{\infty} \mathcal{V}_{n}$ choose an open set $U_{V}$ in $X$ so that $V=E \cap U_{V}$. 
For every $n \geq 1 \quad$ let $\quad \mathcal{G}_{n}=\left(U_{V_{1}} \cap \cdots \cap U_{V_{n}}: V_{1} \in \mathcal{V}_{1}, \ldots, V_{n} \in \mathcal{V}_{n}\right) \quad$ and $G_{n}=\bigcup_{G \in \mathcal{G}_{n}} G$.

Since $X$ is perfect, the set $G_{n}$ is an $F_{\sigma}$-set in $X$. It follows from [4, p. 457] that $G_{n}$ is paracompact space. Then there exists a locally finite in $G_{n} \operatorname{cover} \mathcal{U}_{n}$ of $G_{n}$ by open sets in $G_{n}$, which refines $\mathcal{G}_{n}$. According to Lemma 3.1, there exists a disjoint locally finite in $G_{n}$ cover of $G_{n}$ by ambiguous sets in $G_{n}$, which refines $\mathcal{U}_{n}$. Remove from this cover those sets that do not intersect with $E$ and denote this new system by $\mathcal{W}_{n}$. Let $P_{n}=\cup \mathcal{W}_{n}$. Note that $P_{n} \subseteq G_{n}$. Fix an arbitrary set $W_{n}$ from $\mathcal{W}_{n}$ and denote $W_{n}^{0}=W_{n} \cup\left(X \backslash P_{n}\right)$.

Index the elements of the system $\left\{\mathcal{W}_{n}^{0}\right\} \cup\left(\mathcal{W}_{n} \backslash\left\{\mathcal{W}_{n}\right\}\right)$ and obtain the family $\mathcal{X}_{n}=\left(X_{n, i}: i \in I_{n}\right)$.

Constructed in such a way, the sequence $\left(\mathcal{X}_{n}\right)_{n=1}^{\infty}$ of families $\mathcal{X}_{n}$ satisfies the following properties:

(i) $\quad X=\bigcup_{i \in I} X_{n, i}$;

(ii) $X_{n, i} \cap X_{n, j}=\emptyset, i \neq j$;

(iii) $X_{n, i} \cap E \neq \emptyset$ for all $i \in I_{n}$.

(iv) family $\left(X_{n, i} \cap G_{n}: i \in I_{n}\right)$ is locally finite in $G_{n}$;

(v) $\left|\left\{i \in I_{n} \mid X_{n, i} \backslash G_{n} \neq \emptyset\right\}\right|=1$;

(vi) $\operatorname{diam}\left(X_{n, i} \cap E\right) \leq 1 /\left(2^{n+1}\right)$ for every $i \in I_{n}$.

Since all the elements of the system $\mathcal{W}_{n}$ are ambiguous sets in the open subset $G_{n}$ of a perfect space $X$, all the elements of system $\mathcal{W}_{n}$ are ambiguous sets in $X$. In addition, since $\mathcal{W}_{n}$ is locally finite in $G_{n}$, Michael's theorem [4, p. 430] implies that $P_{n}$ is also an ambiguous set in $X$. This implies that

(vii) $X_{n, i}$ is ambiguous in $X$ for all $i \in I_{n}$.

For every $n \in \mathbb{N}$ let

$$
E_{n, i}=X_{n, i} \cap E
$$

and for all $i_{1} \in I_{1}, \ldots, i_{n} \in I_{n}$ let

$$
\begin{aligned}
& B_{i_{1} \ldots i_{n}}=E_{1, i_{1}} \cap E_{2, i_{2}} \cap \cdots \cap E_{n, i_{n}}, \\
& C_{i_{1} \ldots i_{n}}=X_{1, i_{1}} \cap X_{2, i_{2}} \cap \cdots \cap X_{n, i_{n}} .
\end{aligned}
$$

Then:

(1) $E=\bigcup_{i_{1} \in I_{1}, \ldots, i_{n} \in I_{n}} B_{i_{1} \ldots i_{n}}$ and $X=\bigcup_{i_{1} \in I_{1}, \ldots, i_{n} \in I_{n}} C_{i_{1} \ldots i_{n}}$ for every $n \in \mathbb{N}$;

(2) $\quad B_{i_{1} \ldots i_{n}} \cap B_{j_{1} \ldots j_{n}}=\emptyset$ and $C_{i_{1} \ldots i_{n}} \cap C_{j_{1} \ldots j_{n}}=\emptyset$ if $\left(i_{1}, \ldots, i_{n}\right) \neq\left(j_{1}, \ldots, j_{n}\right)$;

(3) if $m \geq n$ and $C_{i_{1} \ldots i_{n}} \cap C_{j_{1} \ldots j_{m}} \neq \emptyset$ then $i_{1}=j_{1}, \ldots, i_{n}=j_{n}$;

(4) $C_{i_{1} \ldots i_{n}} \cap E=B_{i_{1} \ldots i_{n}}$ for every $n \in \mathbb{N}$ and $i_{1} \in I_{1}, i_{2} \in I_{2}, \ldots, i_{n} \in I_{n}$;

(5) $\quad B_{i_{1} \ldots i_{n}}$ is an ambiguous set in $E$ and $C_{i_{1} \ldots i_{n}}$ is an ambiguous set in $X$ for all $n$ and $i_{1} \in I_{1}, i_{2} \in I_{2}, \ldots, i_{n} \in I_{n}$.

Moreover,

(6) for every $n \in \mathbb{N}$ and any set $I^{\prime} \subseteq I_{1} \times \cdots \times I_{n}$ the set $A=\bigcup_{\left(i_{1}, \ldots, i_{n}\right) \in I^{\prime}} C_{i_{1} \ldots i_{n}}$ is ambiguous in $X$. 
According to (iv), for every $k \in \mathbb{N}$ the family $\left(X_{k, i} \cap G_{k}: i \in I_{k}\right)$ is locally finite in $G_{k}$, and (v) implies that the family $\left(X_{k, i} \cap\left(X \backslash G_{k}\right): i \in I_{k}\right)$ is locally finite in $X \backslash G_{k}$. Therefore, taking into consideration the fact that the sequence $\left(G_{n}\right)_{n=1}^{\infty}$ decreases, we obtain that for an arbitrary set

$$
D \in\left\{X \backslash G_{1}, G_{1} \backslash G_{2}, G_{2} \backslash G_{3}, \ldots, G_{n-1} \backslash G_{n}, G_{n}\right\}=\left\{D_{0}, \ldots, D_{n}\right\}
$$

and for every $k \in\{1,2, \ldots, n\}$ the family $\left(X_{k, i} \cap D: i \in I_{k}\right)$ is locally finite in $D$. Then we have that the family $\left(C_{i_{1}, \ldots, i_{n}}: i_{1} \in I_{1}, \ldots, i_{n} \in I_{n}\right)$ is also locally finite in $D$. Hence, the family $\left(C_{i_{1}, \ldots, i_{n}} \cap D:\left(i_{1}, \ldots, i_{n}\right) \in I^{\prime}\right)$ is locally finite in $D$. Furthermore, since all the sets $D_{0}, \ldots, D_{n}$ are ambiguous in $X$ and (5) holds, all the sets $A_{k}=\bigcup_{\left(i_{1}, \ldots, i_{n}\right) \in I^{\prime}} C_{i_{1}, \ldots, i_{n}} \cap D_{k}$ are ambiguous in $X$ and $A=\bigcup_{k=0}^{n} A_{k}$ is an ambiguous set in $X$.

For every $n$ and $i \in I_{n}$ choose an arbitrary point $y_{n_{i}} \in E_{n_{i}}$. For every $x \in E$ let $\psi_{n}(x)=y_{n, i_{n}}$ if $x \in B_{i_{1} \ldots i_{n}}$. Note that, according to (1) and (2), mappings $\psi_{n}: E \rightarrow E$ are correctly defined. We now show that the sequence $\left(\psi_{n}\right)_{n=1}^{\infty}$ uniformly converges to the identical mapping $\psi: E \rightarrow E, \psi(x)=x$.

Fix $x \in E$ and $n \in \mathbb{N}$. Then there exist $i_{1} \in I_{1}, \ldots, i_{n} \in I_{n}$ such that $x \in B_{i_{1} \ldots i_{n}}$. Then $\psi_{n}(x)=y_{n, i_{n}}$. Since $B_{i_{1} \ldots i_{n}} \subseteq E_{n, i_{n}}, x \in E_{n, i_{n}}$ and $y_{n, i_{n}} \in E_{n, i_{n}}$. According to (vi), we have that $\operatorname{diam} E_{n, i_{n}} \leq 1 /\left(2^{n+1}\right)$. Then

$$
d\left(\psi(x), \psi_{n}(x)\right)=d\left(x, y_{n, i_{n}}\right) \leq \frac{1}{2^{n+1}} .
$$

Note that

$$
d\left(\psi_{m}(x), \psi_{n}(x)\right) \leq \frac{1}{2^{n+1}}+\frac{1}{2^{n+1}}=\frac{1}{2^{n}} \quad \text { for all } m \geq n \text { and } x \in E .
$$

For every $n$ and multi-index $\left(i_{1} \ldots i_{n}\right) \subseteq I_{1} \times \cdots \times I_{n}$ denote

$$
\ell\left(i_{1} \ldots i_{n}\right)=\max \left\{1 \leq k \leq n \mid B_{i_{1} \ldots i_{k}} \neq \emptyset\right\}
$$

For all $n \in \mathbb{N}$ and $x \in X$ let $r_{n}(x)=y_{\ell\left(i_{1} \ldots i_{n}\right), i_{\ell\left(i_{1} \ldots i_{n}\right)}}$ if $x \in C_{i_{1} \ldots i_{n}}$. Properties (1) and (2) imply that all the mappings $r_{n}: X \rightarrow E$ are correctly defined.

We now prove that the sequence $\left(r_{n}\right)_{n=1}^{\infty}$ satisfies inequality $(*)$ for all $x \in X$.

Let $x_{0} \in X$ and $m \geq n$. Then there exist $i_{1} \in I_{1}, \ldots, i_{n} \in I_{n}$ and $j_{1} \in I_{1}, \ldots, j_{m}$ $\in I_{m}$ such that $x_{0} \in C_{i_{1} \ldots i_{n}} \cap C_{j_{1} \ldots j_{m}}$. Property (3) implies that $i_{1}=j_{1}, \ldots, i_{n}=j_{n}$.

If $B_{i_{1} \ldots i_{n}} \neq \emptyset$ then $r_{n}\left(x_{0}\right)=y_{n, i_{n}}$. Let $k=\ell\left(j_{1} \ldots j_{m}\right)$. Then $r_{m}\left(x_{0}\right)=y_{k, j_{k}}$. Clearly, $k \geq n$. Choose any point $x \in B_{j_{1} \ldots j_{k}}$. Since $B_{j_{1} \ldots j_{k}}=B_{i_{1} \ldots i_{n} j_{n+1} \ldots j_{k}} \subseteq B_{i_{1} \ldots i_{n}}$, $\psi_{n}(x)=y_{n, i_{n}}$ and $\psi_{k}(x)=y_{k, j_{k}}$. Inequality $(*)$ implies that

$$
d\left(r_{n}\left(x_{0}\right), r_{m}\left(x_{0}\right)\right)=d\left(y_{n, i_{n}}, y_{k, j_{k}}\right)=d\left(\psi_{n}(x), \psi_{k}(x)\right)<\frac{1}{2^{n}} .
$$

If $B_{i_{1} \ldots i_{n}}=\emptyset$ then $\ell\left(i_{1} \ldots i_{n}\right)=\ell\left(j_{1} \ldots j_{m}\right)$. Now we have that $r_{n}\left(x_{0}\right)=r_{m}\left(x_{0}\right)$ and $d\left(r_{n}\left(x_{0}\right), r_{m}\left(x_{0}\right)\right)=0$. Hence, sequence $\left(r_{n}\right)_{n=1}^{\infty}$ satisfies $(*)$ for all $x \in X$. 
Since $X$ is a completely metrizable space, there exists a mapping $r: X \rightarrow E$ such that the sequence $\left(r_{n}\right)_{n=1}^{\infty}$ uniformly converges to $r$ on $X$. Moreover, since $\left.r_{n}\right|_{E}=\psi_{n}$ and $\psi(x)=\lim _{n \rightarrow \infty} \psi_{n}(x)$ for all $x \in E$, we have that $\left.r\right|_{E}=\psi$, that is $r(x)=x$ for every $x \in E$.

Since a uniform limit of the Lebesgue class one mappings is a Lebesgue class one mapping [12, p. 395], it remains to prove that $r_{n} \in H_{1}(X, E)$ for all $n \in \mathbb{N}$.

Since for any $n \in \mathbb{N}$ and $i_{1}, \ldots, i_{n} \in I_{1} \times \cdots \times I_{n}$ such that $C_{i_{1}, \ldots, i_{n}} \neq \emptyset$ the mapping $\left.r_{n}\right|_{C_{i_{1}, \ldots, i_{n}}}$ is constant, we have that for an arbitrary set $B \subseteq E$

$$
r_{n}^{-1}(B)=\bigcup_{\left(i_{1}, \ldots, i_{n}\right) \in I^{\prime}} C_{i_{1}, \ldots, i_{n}}
$$

where $I^{\prime}=\left\{\left(i_{1}, \ldots, i_{n}\right) \in I_{1} \times \cdots \times I_{n} \mid r_{n}\left(C_{i_{1}, \ldots, i_{n}}\right) \subseteq B\right\}$. Therefore, according to $(6), r_{n}^{-1}(B)$ is an ambiguous set in $X$. In particular, all the mappings $r_{n}$ are of the first Lebesgue class.

Corollary 3.3. Let $X$ be a completely metrizable space and $E \subseteq X$. The set $E$ is an $H_{1}$-retract of $X$ if and only if $E$ is $G_{\delta}$ in $X$.

PROOF. Sufficiency. This immediately follows from 2.3.

Necessity. According to the Aleksandrov-Hausdorff theorem [4, p. 407], the space $E$ is completely metrizable. Hence, Theorem 3.2 implies that $E$ is an $H_{1}$-retract of $X$.

The following corollary gives a positive answer to Question 1.2.

COROllary 3.4. Let $X$ be a metrizable space, $Y$ be a completely metrizable space, $A \subseteq X$ and $f: A \rightarrow Y$ be a continuous mapping. Then there exists a Lebesgue class one mapping $g: X \rightarrow Y$ such that $\left.g\right|_{A}=f$.

Proof. Denote by $\widehat{X}$ the completion of $X$. According to [4, p. 405], there exists a $G_{\delta^{-}}$ subset $\widehat{A}$ of $\widehat{X}$ and a continuous mapping $h: \widehat{A} \rightarrow Y$ such that $A \subseteq \widehat{A}$ and $\left.h\right|_{A}=f$. According to Corollary $3.3, \widehat{A}$ is an $H_{1}$-retract of $\widehat{X}$. Then there exists a mapping $\widehat{h} \in H_{1}(\widehat{X}, Y)$ such that $\left.\widehat{h}\right|_{\widehat{A}}=h$.

Let $g=\left.\widehat{h}\right|_{X}$. Then $g: X \rightarrow Y$ is the desired extension of $f$.

Since every completely metrizable separable space is hereditarily Baire and Lindelöf, the result of Kalenda and Spurný implies the following fact.

THEOREM 3.5. Let E be a completely metrizable separable subspace of a completely regular space $X$. Then $E$ is an $H_{1}$-retract of $X$.

At first sight this theorem gives a solution to the problem of the extension of a continuous mapping to a mapping of the first Lebesgue class with values in an arbitrary (not necessary separable) topological space, analogously to as in Theorem 3.2. However, since a continuous image of a separable space $E$ is also separable, in fact 
separability of $Y$ is present here imperceptibly and we cannot obtain Corollary 3.4 from Theorem 3.5.

The following example shows that the assumption that $X$ is perfect in Theorem 3.2 and the assumption that $E$ is separable in Theorem 3.5 cannot be omitted. Moreover, this example gives a negative answer to Question 1.4.

EXAMPLE 3.6. There exist a completely metrizable subspace $E$ of a compact space $X$ and a continuous function $f: E \rightarrow[0,1]$ which cannot be extended to a Lebesgue class one function on $X$.

ProOf. Let $E$ be an uncountable discrete space and $X=\alpha E=E \cup\{\infty\}$ be the Aleksandrov compactification of $E$.

Choose two uncountable disjoint subsets $E_{1}$ and $E_{2}$ of $E$ so that $E=E_{1} \sqcup E_{2}$ and consider the function

$$
f(x)= \begin{cases}1 & \text { if } x \in E_{1} \\ 0 & \text { if } x \in E_{2}\end{cases}
$$

The function $f: E \rightarrow[0,1]$ is continuous and hence a $\sigma$-discrete function of the first Lebesgue class.

Note that for every continuous function (and for every Baire one function that is a pointwise limit of continuous functions) $g: X \rightarrow[0,1]$ there exists at most countable set $X_{0} \subseteq X$ such that $g(x)=g(\infty)$ for all $x \in X \backslash X_{0}$. It follows that a function $f$ cannot be extended to a Baire one function $g: X \rightarrow[0,1]$, provided $E_{1}$ and $E_{2}$ are uncountable sets.

According to [15, Theorem 3.7], the class $H_{1}(X,[0,1])$ coincides with the class of all Baire one functions $g: X \rightarrow[0,1]$. Therefore, the function $f$ cannot be extended to a Lebesgue class one function on $X$.

\section{Acknowledgement}

The author would like to thank Professor V. Mykhaylyuk for helpful suggestions and comments.

\section{References}

[1] G. Alexits, 'Über die Erweiterung einer Baireschen Funktion', Fund. Math. 15 (1930).

[2] K. Borsuk, Theory of Retracts (Mir, Moscow, 1971), 292 pp (in Russian).

[3] J. Dugundji, 'An extension of Tietze's theorem', Pacific J. Math. 1 (1951), 353-367.

[4] R. Engelking, General Topology (Mir, Moscow, 1986), 752 pp (in Russian).

[5] H. Hahn, Theorie der Reellen Funktionen (Springer, Berlin, 1921).

[6] R. Hansell, 'On Borel mappings and Baire functions', Trans. Amer. Math. Soc. 194 (1974), $195-211$.

[7] - 'Sums, products and continuity of Borel maps', Proc. Amer. Math. Soc. 104(2) (1988), $465-471$.

[8] F. Hausdorff, Set Theory (Moscow, Leningrad, 1934).

[9] O. Kalenda and J. Spurný, 'Extending Baire-one functions on topological spaces', Topology Appl. 149 (2005), 195-216. 
[10] O. Karlova, 'The first functional Lebesgue class and Baire classification of separately continuous mappings', in Naukovyj Visnyk Chernivets'koho Universytetu: Matematyka, 191-192 (Ruta, Chernivtsi, 2004), pp. 52-60 (in Ukrainian).

[11] K. Kuratowski, 'Sur les théorèmes topologiques de la théorie des fonctions de variables réelles', C. R. Acad. Paris 197 (1933), 1090-1091.

[12] - Topology (Mir, Moscow, 1966), 596 pp (in Russian).

[13] W. Sierpińsky, 'Sur l'extension des fonctions de Baire définities sur les ensembles linéaires quelconques', Fund. Math. 16 (1930), 81-89.

[14] A. H. Stone, 'On $\sigma$-discreteness and Borel isomorphism', Amer. J. Math. 85 (1962), 655-666.

[15] L. Veselý, 'Characterization of Baire-one functions between topological spaces', Acta Univ. Carolin. Math. Phys. 33(2) (1992), 143-156.

OLENA KARLOVA, Department of Mathematical Analysis, Chernivtsi National University, Kotsjubyns'koho 2, Chernivtsi 58012, Ukraine

e-mail:mathan@ukr.net 